\title{
Les facteurs explicatifs de l'engagement environnemental des PME dans le secteur de l'agroalimentaire : une étude comparative Canada-France-Finlande
}

\author{
Yves Robichaud, Université Laurentienne (Canada) - Corinna Stocky, Institut Polytechinque \\ LaSalle Beauvais (France) - Nicolas Legrand, Turku University of Applied Sciences (Finlande) \\ et Caroline Godard, Agro-Transfert Ressources et Territoires (France)
}

\section{INTRODUCTION}

\section{L'engagement environnemental, autrefois réservé exclusivement aux environnementalistes s'est taillé une place de choix au sein des dimensions politiques, économiques et sociales dans la majorité des pays développés.}

L'engagement environnemental, autrefois réservé exclusivement aux environnementalistes s'est taillé une place de choix au sein des dimensions politiques, économiques et sociales dans la majorité des pays développés. Il est également bien connu que les gouvernements jouent un rôle important dans cet engagement environnemental mais la responsabilité des entreprises n'est certes pas négligeable étant donné l'influence qu'elles exercent sur l'écologie. À ce sujet, il est mentionné que près de la moitié des émissions de tous les gaz à effet de serre (GES) peuvent être imputés aux opérations des sociétés ${ }^{1}$.

Les petites et moyennes entreprises (PME) ne font pas exception à la règle et sont souvent citées comme l'une des sources polluantes majeures. Il suffit de constater la contribution des PME au sein de plusieurs économies occidentales pour réaliser l'ampleur des conséquences environnementales néfastes qu'elles peuvent causer. Certains auteurs estiment que le bilan environnemental des PME pourrait être responsable jusqu'à $70 \%$ de la pollution totale et qu'il pourrait même surpasser l'impact environnemental combiné des grandes entreprises ${ }^{2}$.

Les PME comptent pour plus de $95 \%$ des entreprises du secteur privé dans la plupart des économies industrialisées ${ }^{3}$. Le secteur des PME en Finlande compte environ 214000 entreprises soit $99,7 \%$ de l'ensemble des entreprises finlan- daises tandis que ce chiffre se situe à près de 2400000 en France soit $99,8 \%$ de toutes les entreprises françaises, soit le même pourcentage de petites entreprises que compte l'Union Européenne ${ }^{4}$. Au Canada, on dénombre environ 2 millions de PME pour $98,8 \%$ des entreprises canadiennes $^{5}$. Dans les trois pays, les PME emploient à elles seules environ six travailleurs sur dix ${ }^{4-5}$. En raison du grand nombre de PME et de sa répercussion néfaste sur l'environnement par la production d'agents polluants et de déchets, la gestion environnementale des PME est incontournable et mérite une attention particulière.

C'est dans cette perspective que cette étude vise à acquérir une meilleure compréhension de l'engagement environnemental au sein des PME canadiennes, finlandaises et françaises qui œuvrent dans le domaine agroalimentaire. Plus spécifiquement, il s'agit d'abord de voir si certains facteurs internes (facteurs liés aux ressources, aux compétences et aux connaissances) sont susceptibles de favoriser l'engagement environnemental. Il sera aussi question d'identifier les obstacles qui empêchent les dirigeants de PME de s'engager davantage en matière d'environnement. Une meilleure compréhension de ces facteurs et de ces obstacles permettra d'identifier et de comprendre les aspects à améliorer pour les PME ainsi que pour les agences gouvernementales qui doivent déployer les politiques en matière de gestion de l'environnement dans le milieu des entreprises.

Le bilan environnemental des PME pourrait être responsable jusqu'à $70 \%$ de la pollution totale et qu'il pourrait même surpasser l'impact environnemental combiné des grandes entreprises. 
Pour y arriver, nous aurons recours à un échantillon de 101 PME canadiennes, françaises et finlandaises opérant dans le secteur agroalimentaire $^{\mathrm{a}}$. Le modèle retenu pour cette étude s'inspire en majeure partie de celui de Roy, Berger-Douce, et Audet ${ }^{6}$. Cependant, notre étude se démarque de l'étude de Roy, Berger-Douce, et Audet $^{6}$ et des autres études sur le sujet, en ciblant uniquement le domaine agroalimentaire, en incorporant au modèle la variable « obstacles à l'engagement environnemental » et en comparant les résultats obtenus des trois pays parti-

\section{REVUE DE LITTÉRATURE}

Diverses études consultées ${ }^{6,7,8,9,10,11}$ indiquent qu'avant 2000, les entreprises en général ne se préoccupaient guère de l'engagement environnemental. Les dirigeants de PME étaient décrits à cette époque ${ }^{2}$ comme des personnes ignorantes des impacts environnementaux de leur entreprise, sceptiques à propos des avantages liés à une pratique environnementale plus saine, résistants à entreprendre des actions environnementales faute de temps et de ressources financières et, enfin, non pourvus en termes d'outils et de ressources pour faire face aux problèmes environnementaux.

Cependant, des recherches plus récentes suggèrent un changement d'attitude de la part des dirigeants d'entreprises au cours de la dernière décennie $^{6,8,12}$. En effet, de plus en plus d'entreprises intègrent graduellement des préoccupations environnementales dans leur stratégie d'entreprise. À titre d'exemple, une étude ${ }^{12}$ indique que $46 \%$ des PME du Royaume-Uni utilisent des pratiques environnementales et que $35 \%$ d'entre elles ont une politique environnementale en place.

Une autre étude ${ }^{6}$ rapporte le même constat en affirmant que $86 \%$ des 4434 dirigeants de PME interrogés en France considèrent le management environnemental comme une de leurs principales préoccupations. Finalement, une étude récente ${ }^{8}$ rapporte une nette amélioration des pratiques environnementales des PME du Royaume-Uni. En effet, dans cette étude menée auprès de 220 PME de la région de Londres, les trois-quarts des répondants se sont dits en désaccord avec le fait que la taille de leur entreprise soit trop petite cipants à l'étude ${ }^{\mathrm{b}}$. À notre connaissance, aucune étude comparative n'a déjà été menée sur le domaine agroalimentaire ce qui justifie amplement la réalisation de cette étude.

L'article est structuré de la façon suivante : la première partie présente une revue de la littérature ainsi que le cadre conceptuel retenu. En second lieu, la méthodologie utilisée dans l'étude est décrite tandis que les résultats de l'étude et une discussion des points marquants sont présentés dans la dernière partie de l'article.

pour avoir un impact environnemental. Ces quelques exemples semblent démontrer que l'on accorde plus d'importance à l'engagement environnemental qu'on ne le faisait auparavant et que les PME sont plus sensibilisées aux enjeux du management environnemental.

\subsection{Principaux obstacles à l'engagement environnemental}

Malgré cette sensibilisation accrue de la part des PME à l'importance du management environnemental, il existe encore plusieurs obstacles qui freinent un engagement accru des PME dans ce domaine. Parmi les obstacles identifiés à ce jour, un manque de ressources au niveau financier serait l'entrave majeure à l'engagement environ-

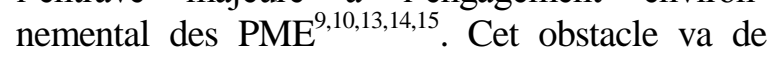
pair avec un certain scepticisme de la part de plusieurs dirigeants de PME quant aux retombées réelles que peut engendrer une pratique écologique plus saine, car cette pratique, selon eux ${ }^{10,16,17}$, peut exiger des coûts supplémentaires élevés.

Au-delà de l'aspect purement économique des ressources, il y a aussi le manque de temps dédié aux causes écologiques ${ }^{17,18}$ et le manque de sensibilisation des PME à l'égard de l'envergure des impacts de leurs activités sur l'environnement ${ }^{19,20}$. De plus, plusieurs PME ne s'intéressent pas à cette préoccupation tant qu'il n'y a pas d'obligations ou d'incitatifs réglementaires. En effet, plusieurs études révèlent que les PME sont peu susceptibles d'adopter volontairement des pratiques environnementales à moins d'y être forcées ${ }^{8,16,21}$. 
D'autres freins à l'engagement environnemental sont aussi rapportés dans la littérature sur les PME, notamment un manque d'information sur les programmes d'aide à l'environnement ${ }^{22}$, un manque de connaissances des problèmes environnementaux $^{11}$ et le fait que les outils de management environnemental existants seraient conçus pour et par les grandes entreprises et non selon les spécificités des PME, ce qui compliquerait leur transfert dans le contexte de $\mathrm{PME}^{16}$. Enfin, les caractéristiques personnelles telles que l'âge, le genre et le niveau d'éducation seraient des facteurs qui influenceraient les valeurs personnelles du dirigeant en termes de protection de l'environnement ${ }^{7,23}$.

\subsection{Le cadre conceptuel proposé}

Tel que mentionné au début de l'article, un des objectifs de l'étude est de vérifier si certains facteurs internes de l'entreprise sont susceptibles d'encourager l'engagement environnemental des PME. Pour y arriver, nous nous sommes largement inspirés de la littérature récente ${ }^{6}$ qui regroupe les facteurs internes retenus pour l'étude en trois catégories distinctes: les ressources, les compétences et les connaissances. Afin de compléter le cadre conceptuel, nous avons ajouté les obstacles perçus par les dirigeants d'entreprise comme un frein à un engagement environnemental. L'ajout de cette variable nous apparaît important dans la mesure où la compréhension des obstacles auxquels se heurtent les dirigeants de PME représente l'un des principaux domaines d'étude du management environnemental au cours des dernières années. Les nombreux travaux répertoriés sur les obstacles perçus par les PME en témoignent ${ }^{8,10}$. La figure 1 présente les composantes du cadre conceptuel de recherche.

Figure 1 - Cadre conceptuel de l'étude proposée

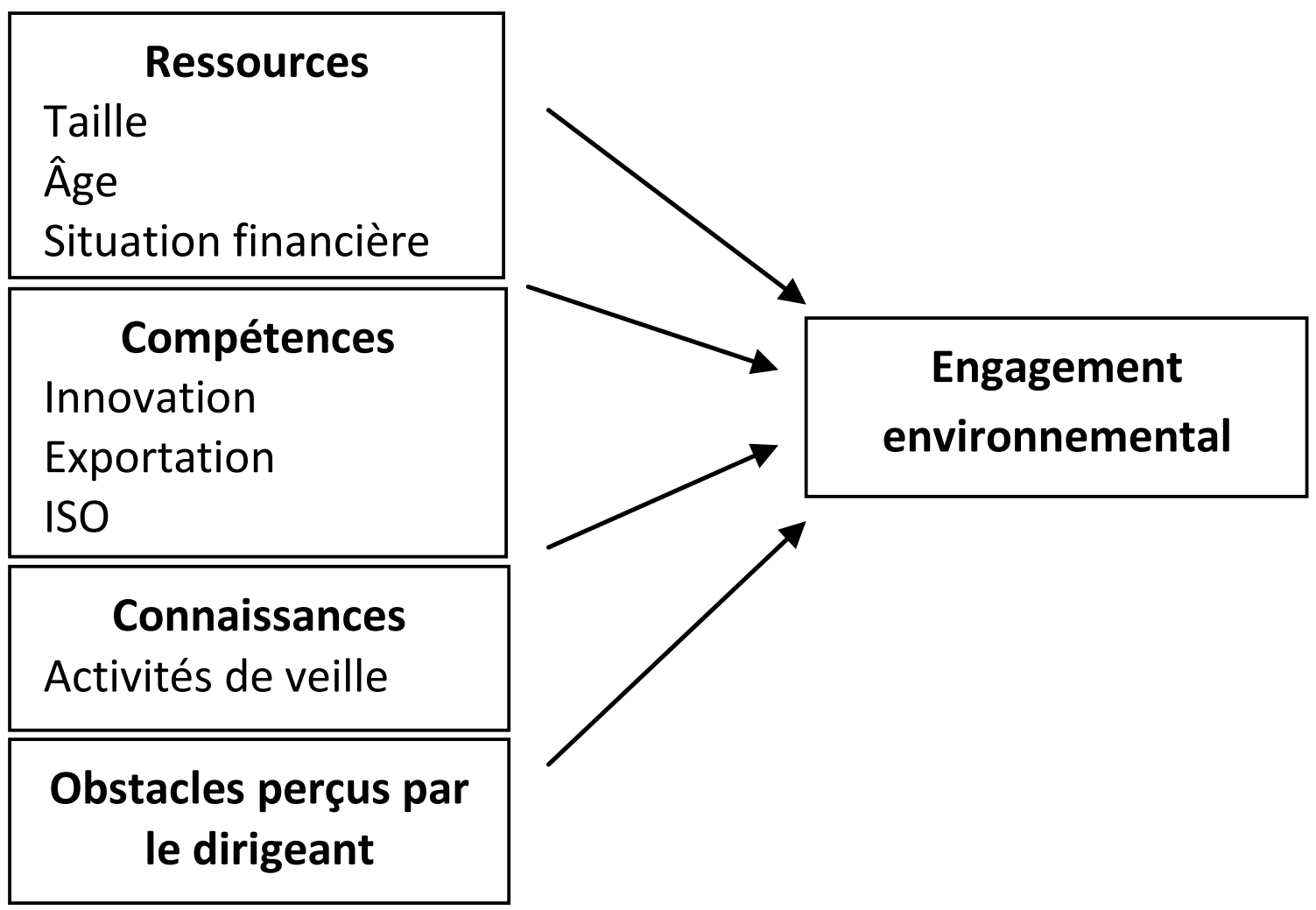


La dimension ressources est composée de la taille de l'entreprise, son âge et sa situation financière. $\mathrm{Au}$ sujet de la taille de l'entreprise, il apparait que les dirigeants des petites entreprises (moins de cinq employés) considéraient l'impact environnemental sur leurs entreprises comme étant négligeable. De plus, plusieurs des obstacles à l'engagement environnemental devenaient encore plus importants à mesure que la taille de l'entreprise diminuait $^{24}$. En outre, dans une autre étude, la taille de l'entreprise a été identifiée comme un facteur majeur dans l'engagement environnemental des entreprises interrogées ${ }^{12}$.

\section{Le manque de ressources financières serait l'entrave majeure à l'engagement environnemental des PME. Dans la littérature il a identifié une tendance qui démontre l'existence d'un lien positif entre la performance environnementale des entreprises et leur situation financière.}

Tel que nous l'avons souligné dans la discussion sur les obstacles à un engagement environnemental, le manque de ressources financières serait l'entrave majeure à l'engagement environnemental des PME. Dans la littérature il a identifié une tendance qui démontre l'existence d'un lien positif entre la performance environnementale des entreprises et leur situation financière ${ }^{10}$.

Quant aux entreprises plus âgées, le fait que certaines études ont établi un lien entre la performance financière et l'âge des entreprises ${ }^{25}$ et que la performance financière semble être liée à un meilleur engagement environnemental ${ }^{10}$, il

\section{MÉTHODOLOGIE}

\subsection{Définition d'une PME}

\author{
Afin de faciliter les comparaisons entre le \\ Canada, la France et la Finlande, la \\ définition de PME de l'Organisation de \\ Coopération et de Développement \\ Économiques (OCDE) a été retenue soit \\ une entreprise de moins de 250 employés.
}

semble donc légitime de penser qu'une entreprise plus âgée pourrait être associée à un engagement environnemental accru.

La dimension compétences du modèle comprend l'innovation, l'exportation et la gestion de la qualité (certification ISO). Les recherches répertoriées sur ces trois variables ${ }^{6}$ démontrent $^{2}$ que les entreprises innovantes, exportatrices et dotées d'un programme ISO seraient plus actives en matière d'environnement.

Quant à la dimension connaissances, elle peut se définir comme les connaissances qu'une entreprise accumule au fil de son expérience par l'entremise de diverses sources. Un système formel ou informel d'acquisition et de gestion des connaissances organisationnelles est primordial avant d'entreprendre toute démarche sérieuse en matière d'engagement environnemental. Ce constat est d'autant plus vrai que l'on reproche fréquemment aux dirigeants de PME d'être mal informés à l'égard d'initiatives vertes telles que les technologies disponibles, les lois applicables et les programmes d'aide ${ }^{6,7,11}$.

\section{Un système formel ou informel d'acquisition et de gestion des connaissances organisationnelles est primordial avant d'entreprendre toute démarche sérieuse en matière d'engagement environnemental. Une limite inférieure de 20 employés a aussi été établie arbitrairement afin de s'assurer que les entreprises participantes aient un minimum d'activités liées à la gestion environnementale.}

Afin de faciliter les comparaisons entre le Canada, la France et la Finlande, la définition de PME de l'Organisation de Coopération et de Développement Économiques (OCDE) a été retenue soit une entreprise de moins de 250 employés. Une limite inférieure de 20 employés a aussi été établie arbitrairement afin de s'assurer que les entreprises participantes aient un minimum d'activités liées à la gestion environnementale. 


\subsection{Population et échantillonnage}

L'échantillon des entreprises canadiennes est formé de 1095 entreprises du secteur agroalimentaire provenant d'une banque de données obtenue d'Info-Canada. La Finlande a eu recours aux annuaires d'entreprises des Chambres de commerce des villes les plus importantes (Helsinki, Turku et Tamperee) ainsi qu'à une association de l'industrie agroalimentaire de la Finlande pour un total de 250 entreprises. Quant à la France, elle a eu recours aux deux bases de données suivantes: un fichier national des entreprises de la Chambre de Commerce et d'Industrie et une base de données d'entreprises nationales de LaSalle Beauvais pour un total de 180 entreprises.

Une fois les listes d'entreprises obtenues, des appels téléphoniques ont été effectués, ou des courriels envoyés aux dirigeants des entreprises, afin de leur expliquer le but de l'étude, et de leur demander d'y participer. Dans le cas de la Finlande, les questionnaires ont été envoyés par courriel aux dirigeants ayant accepté de participer à l'étude tandis que pour la France et le Canada, les entrepreneurs étaient invités à remplir le questionnaire au moyen du logiciel Sphinx sur internet. Des rappels hebdomadaires ont aussi été effectués dans le but d'inciter les entrepreneurs à participer à l'étude.

Sur le total de 1095 entreprises canadiennes, 459 n'ont pu être jointes après trois rappels effectués. Sur les 636 entreprises qui ont accepté de participer à l'étude, 43 questionnaires ont été reçus, soit un taux de réponse de $6,7 \%$ (43/636). La Finlande a connu plus de succès avec 42 questionnaires complétés sur un envoi de 250 questionnaires, soit un taux de réponse de $16,8 \%$ tandis que la France a reçu 16 questionnaires (taux de réponse de 8,8\%) sur un total de 180 questionnaires envoyés.

Au total, 101 questionnaires ont été reçus. Pour expliquer le faible taux de réponse obtenu dans notre étude ${ }^{26}$, Macpherson et Wilson soutiennent qu'il est très difficile de convaincre les dirigeants de PME de participer à des études de ce genre et qu'il est courant de voir des taux de réponse faibles dans les recherches sur les PME.

\subsection{Instrument de mesure}

Le questionnaire ${ }^{6}$ utilisé dans notre étude est celui développé par une équipe de chercheurs auquel nous avons ajouté une question sur les obstacles. La question sur les obstacles a été développée à partir des réponses obtenues lors d'un atelier de travail sur la gestion environnementale auquel une vingtaine de dirigeants de PME canadiens ont participé. À l'exception des variables âge, taille, exportation et certification ISO, des échelles de type Likert (de $1=$ aucune importance à $5=$ très grande importance) ont été utilisées. Les variables de l'étude sont regroupées en fonction du modèle présenté à la figure 1 dans la section « revue de littérature ».

Le tableau 1 qui suit présente ces variables ainsi que les renseignements relatifs à la fiabilité des construits : construit lié à la situation financière (4 éléments), construit lié à l'innovation (4 éléments) et construit lié à l'engagement environnemental (4 éléments). La fiabilité des construits a été évaluée à l'aide de l'alpha de Cronbach. À cet effet, les résultats sur la fiabilité des construits se sont révélés excellents avec des coefficients de 0,867, 0,851 et 0,885 respectivement pour les construits situation financière, innovation et engagement environnemental. Pour un instrument composé de petites échelles de 3 à 4 énoncés, un coefficient alpha de 0,70 et plus est jugé satisfaisant et indique que les échelles semblent mesurer le même construit ${ }^{27}$.

Finalement, afin de mesurer l'engagement environnemental des répondants, nous avons utilisé la moyenne des quatre éléments constituant la dimension engagement environnemental pour créer deux groupes de références : faible engagement environnemental et fort engagement environnemental selon que la valeur de l'engagement environnemental était supérieure ou inférieure à la moyenne. 


\section{Tableau 1 - Variables de l'étude}

\begin{tabular}{|c|c|}
\hline Variables & Mesure \\
\hline \multicolumn{2}{|l|}{ Ressources } \\
\hline Taille & Nombre d'employés \\
\hline Âge & Nombre d'années depuis la création \\
\hline Situation financière & Moyenne de 4 éléments (échelle de type Likert) \\
\hline \multirow[t]{4}{*}{$($ alpha de Cronbach $=0.867)$} & 1. Croissance des ventes \\
\hline & 2. Bénéfices \\
\hline & 3. Rendement sur les ventes \\
\hline & 4. Rendement sur investissement \\
\hline \multicolumn{2}{|l|}{ Compétences } \\
\hline Innovation & Moyenne de 4 éléments (échelle de type Likert) \\
\hline \multirow[t]{4}{*}{ (alpha de Cronbach $=0.851)$} & 1. Innovation sur les produits \\
\hline & 2. Innovation sur les procédés de fabrication \\
\hline & 3. Investissements en R\&D \\
\hline & 4. Introduction de nouveaux produits-services \\
\hline Exportation & \% régional, national, international \\
\hline Certification ISO & Variable dichotomique (oui ou non) \\
\hline \multicolumn{2}{|l|}{ Connaissances } \\
\hline \multirow[t]{10}{*}{ Activités de veille environnementale } & 10 éléments (échelle de type Likert) \\
\hline & 2. Communication avec laboratoires de recherche et universités \\
\hline & 3. Communication avec associations industrielles \\
\hline & 4. Communication avec consultants \\
\hline & 5. Communication avec agences gouvernementales \\
\hline & 6. Suivi des changements du marché \\
\hline & 7. Suivi des changements technologiques \\
\hline & 8. Communication avec clients \\
\hline & 9. Communication avec concurrents \\
\hline & 10. Communication avec fournisseurs \\
\hline \multirow[t]{5}{*}{$\begin{array}{l}\text { Engagement environnemental } \\
\text { (alpha de Cronbach }=0.885 \text { ) }\end{array}$} & Moyenne de 4 éléments (échelle de type Likert) \\
\hline & 1. Fort intérêt dans la gestion des aspects environnementaux \\
\hline & 2. Instaure activement des solutions aux problèmes environnementaux \\
\hline & 3. Politique environnementale écrite et détaillée \\
\hline & 4. Engagement au-delà de la conformité aux réglementations \\
\hline \multirow{13}{*}{ Obstacles } & \\
\hline & 10 éléments (échelle de type Likert) \\
\hline & 1. Coûts élevés \\
\hline & 2. Nécessite trop de temps et d'effort \\
\hline & 3. Manque de sensibilisation et de connaissances de la part des dirigeants \\
\hline & 4. Perception qu'un expert est requis et que le coût associé est trop élevé \\
\hline & 5. Perception de retombées minimes sur l'investissement à court terme \\
\hline & 6. Nécessite des contrôles additionnels \\
\hline & 7. Nécessite de la documentation additionnelle \\
\hline & 8. Peur, hostilité et crainte associées avec l'attitude du management face \\
\hline & à des pratiques d'affaires plus respectueuses de l'environnement \\
\hline & 9. Aucun encouragement ou incitatif réglementaire \\
\hline & 10. Autres \\
\hline
\end{tabular}




\subsection{Traitement et analyse des données}

Le traitement statistique des données a été fait à l'aide du logiciel «Statistical Program for Social Sciences » (SPSS). Des tests en $t$ de Student ont été effectués pour comparer les résultats obtenus de chaque pays sur les variables ressources, compétences, connaissances, obstacles et afin d'évaluer l'ensemble des résultats sur la base de leur niveau d'engagement environnemental (fort versus faible engagement environnemental).
Les résultats sont présentés dans la prochaine section selon l'ordre suivant: les caractéristiques de l'échantillon sont discutées en premier suivis des différences statistiquement significatives relevées dans la comparaison des résultats entre les trois pays ${ }^{c}$ et des résultats de l'ensemble de l'étude selon leur niveau d'engagement environnemental (faible ou fort).

\section{PRÉSENTATION ET DISCUSSION DES RÉSULTATS}

\subsection{Caractéristiques de l'échantillon}

Le tableau 2 présente les caractéristiques de l'échantillon des 101 PME agroalimentaires. L'âge moyen des PME de l'échantillon est de 30.27 ans avec, en moyenne, 37 employés à leur service. À noter que les PME de la France sont plus âgées et comptent davantage d'employés que celles du Canada et de la Finlande. La situation financière des entreprises participantes est quasi identique entre les trois pays avec des résultats se situant tout près de la moyenne de 3.14. On observe également un plus fort niveau d'engagement environnemental chez les dirigeants français (3.52) que chez les dirigeants canadiens (3.44) et finlandais (2.89).

Tableau 2 - Caractéristiques de l'échantillon par pays

\begin{tabular}{lcccc}
\hline & Moyenne & Canada & Finlande & France \\
\hline $\mathrm{N}=101$ & & 43 & 42 & 16 \\
Age & 30.27 & 26.86 & 29.90 & 42.46 \\
Taille & 37.33 & 36.12 & 31.81 & 65.70 \\
Situation financière & 3.14 & 3.08 & 3.22 & 3.10 \\
Engagement environnemental & 3.23 & 3.44 & 2.89 & 3.52 \\
\hline
\end{tabular}

\subsection{Résultats - comparaison entre les trois pays}

L'analyse des résultats comparatifs entre les trois pays révèle peu de différences statistiquement significatives (voir tableau 3). La première différence observée porte sur la variable engagement environnemental où les dirigeants des PME canadiennes (3.44) et des PME françaises (3.52) disent avoir un plus haut niveau d'engagement environnemental que les dirigeants de la Finlande (2.89).

Au niveau de l'acquisition de connaissances, on remarque une plus grande activité en recherche et développement $(\mathrm{R} \& \mathrm{D})$ de la part des PME finlandaises (2.86) en comparaison avec la France (2.06) et le Canada (2.67). En France, une enquête ${ }^{28}$ menée auprès de 1700 Petites et
Moyennes Industries (PMI) de l'agroalimentaire en 2008-2009 confirme que ces entreprises souffrent d'un certain retard dans le domaine de la R\&D (cité par elles à $43 \%$ comme moyen de mise en place du développement durable, contre $53 \%$ pour toutes les PME confondues). Ce domaine de la $R \& D$ dans les PMI agroalimentaires françaises mobilise en effet des investissements limités et concerne très majoritairement les procès de fabrication au détriment des produits, ce qui n'en fait pas un moyen privilégié pour ces entreprises pour mettre en place des mesures favorables à l'environnement. De plus, les dirigeants des PME de France privilégient davantage une communication avec les agences gouvernementales comme source d'information que les dirigeants de la Finlande qui, par contre, 
consultent davantage leurs clients, alors que les dirigeants canadiens utilisent les deux sources d'information (agences gouvernementales et clients).

Finalement, le seul obstacle qui ressort statistiquement significatif de l'analyse des résultats est l'attitude du management vis-à-vis des pratiques d'affaires plus respectueuses de l'environnement où les dirigeants canadiens perçoivent ce facteur comme étant un obstacle plus important à un plus grand engagement environnemental que leurs homologues finlandais.

\section{Tableau 3 - Comparaison entre pays (Résultats significatifs seulement)}

\begin{tabular}{|c|c|c|c|c|c|c|c|c|c|}
\hline & \multicolumn{3}{|c|}{ Pays } & \multicolumn{6}{|c|}{ Niveau de signification $\left(p^{a}\right)$} \\
\hline & Canada & Finlande & France & Can & & Fin-1 & & Can-F & \\
\hline \multicolumn{10}{|c|}{ Stratégie environnementale et niveau d'engagement } \\
\hline Engagement environnemental & 3.44 & 2.89 & 3.52 & 0.004 & $* * *$ & 0.075 & $*$ & 0.828 & \\
\hline \multicolumn{10}{|l|}{ Acquisition de connaissances } \\
\hline $\begin{array}{l}\text { R-D effectuée par votre } \\
\text { organisation }\end{array}$ & 2.67 & 2.86 & 2.06 & 0.454 & & 0.026 & $* *$ & 0.090 & * \\
\hline $\begin{array}{l}\text { Communication avec agences } \\
\text { gouvernementales }\end{array}$ & 3.07 & 2.43 & 3.06 & 0.002 & $* * *$ & 0.021 & $* *$ & 0.972 & \\
\hline Suivi des changements du marché & 3.40 & 3.00 & 2.81 & 0.024 & $* *$ & 0.543 & & 0.067 & $*$ \\
\hline Communication avec vos clients & 3.33 & 3.48 & 2.56 & 0.480 & & 0.003 & $* * *$ & 0.014 & $* *$ \\
\hline \multicolumn{10}{|c|}{ Obstacles à un plus grand engagement environnemental } \\
\hline $\begin{array}{l}\text { Peur, hostilité et crainte associées } \\
\text { avec l'attitude du management } \\
\text { face à des pratiques d'affaires plus } \\
\text { respectueuses de l'environnement }\end{array}$ & 2.88 & 2.17 & 2.62 & 0.002 & $* * *$ & 0.194 & & 0.474 & \\
\hline
\end{tabular}

\subsection{Résultats - ensemble des échantillons}

Étant donné le peu de différences observées dans l'analyse des résultats comparatifs entre les trois pays, nous avons combiné ensemble les trois échantillons afin de voir les tendances qui se dégagent selon le niveau d'engagement environnemental des entreprises (faible ou fort).

Tel qu'indiqué précédemment, le niveau d'engagement environnemental a été déterminé à partir de la moyenne des quatre énoncés constituant la dimension engagement environnemental. À ce sujet, le tableau 4 révèle qu'il existe des différences significatives $(\mathrm{p}<0.001)$ entre les PME avec un fort niveau d'engagement environnemental et celles avec un faible niveau d'engagement environnemental pour chacun des énoncés pris individuellement et lorsque les quatre énoncés sont regroupés ensemble pour ne former qu'un seul facteur. Dans tous les cas, les résultats favorisent les PME avec un fort engagement environnemental. Il est également intéressant de noter que l'ordre d'importance des 4 énoncés stratégiques est identique peu importe le niveau d'engagement environnemental. Ainsi, l'énoncé fort intérêt dans la gestion des aspects environnementaux arrive au premier rang pour l'ensemble des dirigeants interrogés tandis que l'énoncé politique environnemental écrite et détaillée s'avère le moins important des quatre.

On peut supposer que la raison d'un tel résultat réside dans le fait que les deux premiers énoncés (fort intérêt dans la gestion des aspects environnementaux, instaure activement des solutions aux problèmes environnementaux) sont plus susceptibles d'être favorisés par les dirigeants vu leur accessibilité que les deux derniers qui exigent un effort accru ainsi que des coûts plus élevés. 
Tableau 4 - Stratégie environnementale et niveau d'engagement

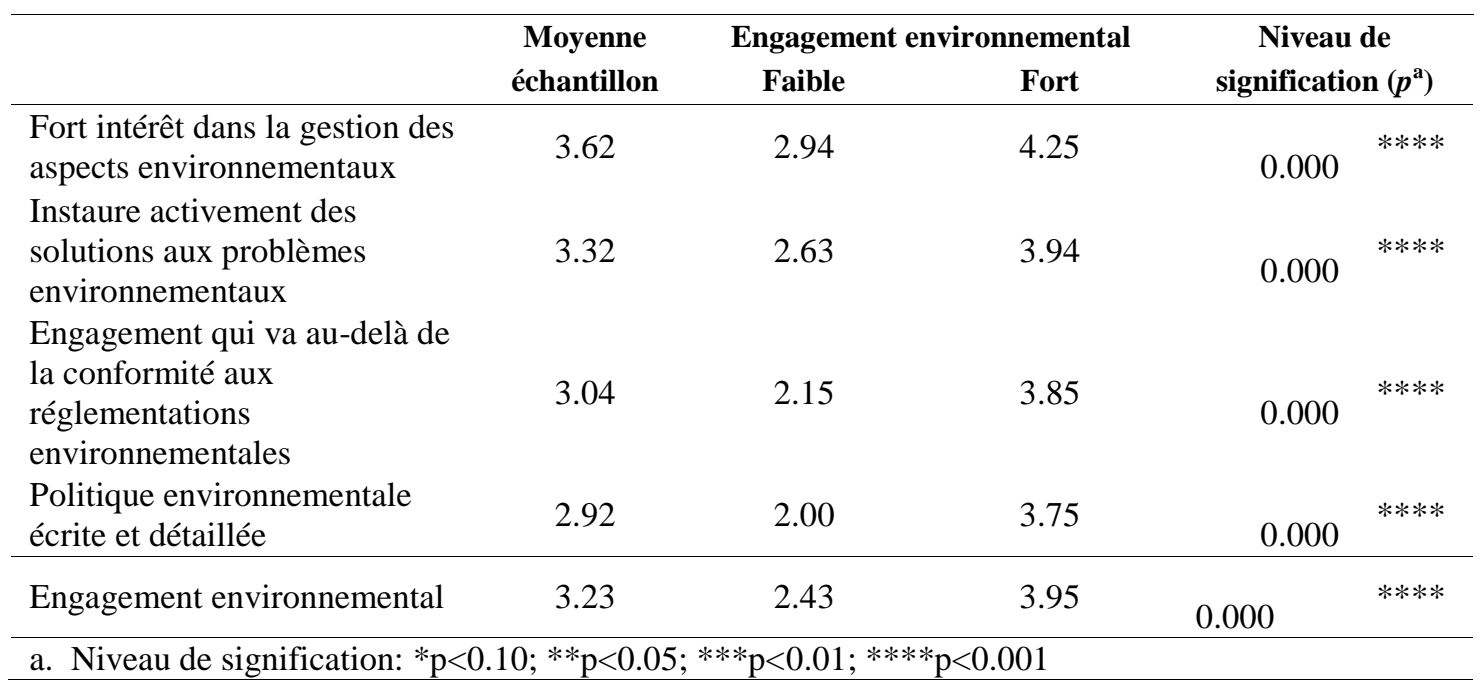

\subsubsection{Ressources}

Le tableau 5 met en valeur les résultats obtenus sur les ressources et l'engagement environnemental. Dans notre étude, les variables constituant la dimension ressources notamment l'âge, la performance financière et la taille de l'entreprise ne semblent pas contribuer à intensifier l'engagement vert au sein des PME agroalimentaires du Canada, de la Finlande et de la France.
En effet, bien que les résultats sur l'âge, la situation financière et la taille soient plus élevés chez les PME avec un engagement environnemental fort, les différences observées ne sont pas statistiquement significatives. Dans une recherche $^{6}$, seule la taille de l'entreprise s'est révélée statistiquement significative $(\mathrm{p}<0.001)$ entre les deux groupes.

\section{Tableau 5 - Ressources et engagement environnemental}

\begin{tabular}{lcccc}
\hline & $\begin{array}{c}\text { Moyenne } \\
\text { échantillon }\end{array}$ & $\begin{array}{c}\text { Engagement } \\
\text { Faible }\end{array}$ & $\begin{array}{c}\text { Environnemental } \\
\text { Fort }\end{array}$ & $\begin{array}{c}\text { Niveau de } \\
\text { signification }\left(p^{\text {a }}\right)\end{array}$ \\
\hline Âge & 30.27 & 30.09 & 30.42 & 0.954 \\
Situation financière & 3.14 & 3.09 & 3.20 & 0.424 \\
Taille & 37.33 & 34.64 & 39.74 & 0.449 \\
\hline a. Niveau de signification: $* \mathrm{p}<0.10 ; * * \mathrm{p}<0.05 ; * * * \mathrm{p}<0.01 ; * * * * \mathrm{p}<0.001$ & \\
\hline
\end{tabular}

\subsubsection{Compétences}

Dans notre étude, la dimension compétences est définie par l'innovation, l'envergure géographique (exportation) et la gestion de la qualité (certification ISO). Les résultats révèlent que l'innovation est positivement associée à un engagement environnemental fort. Dans ce sens, les entreprises démontrant un engagement plus prononcé envers des causes écologiques sont dotées d'une capacité innovatrice accrue $(\mathrm{p}<0.001)$. L'analyse du tableau
6 indique que l'ensemble des entreprises consacre plus de ressources à l'introduction de nouveaux produits ou services tandis que celles présentant un engagement environnemental plus élevé consacrent plus de ressources à l'ensemble des 4 énoncés et, plus spécialement, à l'innovation sur les produits $(\mathrm{p}<0.001)$ et à la recherche et développement $(p<0.001)$. À noter que le facteur innovation a aussi été trouvé significatif $(\mathrm{p}<0.05)$ dans une étude antérieure ${ }^{6}$. 
Tableau 6 - Innovation et engagement environnemental

\begin{tabular}{|c|c|c|c|c|c|}
\hline \multirow[b]{3}{*}{ Innovation sur les produits } & \multirow{3}{*}{$\begin{array}{c}\begin{array}{c}\text { Moyenne } \\
\text { échantillon }\end{array} \\
3.12\end{array}$} & \multicolumn{2}{|c|}{ Engagement environnemental } & \multirow{2}{*}{\multicolumn{2}{|c|}{$\begin{array}{c}\text { Niveau de } \\
\text { signification }\left(p^{\mathrm{a}}\right)\end{array}$}} \\
\hline & & \multirow{2}{*}{$\begin{array}{c}\text { Faible } \\
2.74\end{array}$} & \multirow{2}{*}{$\frac{\text { Fort }}{3.45}$} & & \\
\hline & & & & 0.000 & $* * * *$ \\
\hline $\begin{array}{l}\text { Innovation sur les procédés de } \\
\text { fabrication }\end{array}$ & 3.13 & 2.89 & 3.34 & 0.025 & $* *$ \\
\hline Investissements en $\mathrm{R} \& \mathrm{D}$ & 2.90 & 2.46 & 3.30 & 0.000 & $* * * *$ \\
\hline $\begin{array}{l}\text { Introduction de nouveaux } \\
\text { produits-services }\end{array}$ & 3.32 & 3.13 & 3.49 & 0.067 & $*$ \\
\hline Innovation & 3.12 & 2.81 & 3.40 & 0.001 & $* * * *$ \\
\hline
\end{tabular}

Tableau 7 - Exportation et engagement environnemental

\begin{tabular}{lcccc}
\hline & $\begin{array}{c}\text { Moyenne } \\
\text { échantillon }\end{array}$ & \multicolumn{2}{c}{ Engagement environnemental } & Total \\
\hline Non & $49 \%$ & $51 \%$ & $49 \%$ & $100 \%$ \\
Oui & $51 \%$ & $43 \%$ & $57 \%$ & $100 \%$ \\
\hline Peason Chi-Square: $\mathrm{p}=0.430 ;$ Phi: $-0.079 ;$ Cramer's V: 0.079 & & \\
\hline
\end{tabular}

Les tableaux 7 et 8 présentent les résultats sur l'envergure géographique et la gestion de la qualité. Bien qu'aucun résultat ne ressorte statistiquement significatif, on observe cependant que les entreprises avec un fort engagement environnemental exportent davantage $(57 \%)$ et sont certifiées ISO (63\%).

Tableau 8 - Certification ISO et engagement environnemental

\begin{tabular}{|c|c|c|c|c|}
\hline & \multirow{2}{*}{$\begin{array}{l}\text { Moyenne } \\
\text { échantillon }\end{array}$} & \multicolumn{2}{|c|}{ Engagement environnemental } & \multirow[t]{2}{*}{ Total } \\
\hline & & Faible & Fort & \\
\hline Non & $92 \%$ & $48 \%$ & $52 \%$ & $100 \%$ \\
\hline Oui & $8 \%$ & $38 \%$ & $63 \%$ & $100 \%$ \\
\hline
\end{tabular}

\subsubsection{Connaissances}

En premier lieu, le tableau 9 montre que, lorsqu'il s'agit de s'informer sur le sujet, les PME interrogées privilégient d'abord la communication avec leurs clients (3.27) et leurs fournisseurs (3.20). Ce constat est tout à fait logique si l'on pense aux nombreuses interactions ainsi qu'à la relation d'affaires privilégiée qu'entretiennent les PME avec ces deux groupes. De nombreuses recherches rapportent des résultats similaires. Par exemple, certains chercheurs ${ }^{11}$ ont trouvé des résultats similaires dans la mesure où la communication avec leurs clients est arrivée au premier rang (4.25/5) tandis que la communication avec leurs fournisseurs arrivait au troisième rang $(3.85 / 5)$ après le département interne de recherche et développement. Selon une recherche ${ }^{7}$, les clients et les employés ont été identifiés comme une source d'influence majeure dans l'adoption d'un système de gestion environnemental. Enfin, les résultats obtenus par une étude antérieure vont dans le même sens avec les fournisseurs $(3.15 / 5.00)$ et les clients $(2.83 / 5.00)$ occupant les deux premières positions. À l'autre extrémité, les PME agroalimentaires convoitent moins les laboratoires de recherche et les universités (2.22) ainsi que les 
associations industrielles (2.54) dans leur recherche d'informations ${ }^{6}$.

Les résultats sur les connaissances révèlent également une différence statistiquement significative entre la plupart des activités de veille environnementale et le niveau d'engagement environnemental des entreprises. En effet, les entreprises démontrant un fort engagement environnemental communiquent davantage avec les consultants (3.17), les agences gouvernementales (3.15), les associations industrielles (2.87), les laboratoires de recherche et les universités (2.49) ainsi que le département interne de recherche et développement (2.91) que celles avec un faible engagement environnemental. À l'opposé, la communication avec les clients et les fournisseurs sont les deux groupes où l'on dénote le moins de différences entre les deux niveaux d'engagement environnemental ce qui signifie que ces deux groupes (clients et fournisseurs) constituent des sources d'information importantes pour l'ensemble des dirigeants interrogés.

\section{Tableau 9 - Intensité des activités de veille environnementale et engagement environnemental}

\begin{tabular}{lccccc}
\hline & $\begin{array}{c}\text { Moyenne } \\
\text { échantillon }\end{array}$ & $\begin{array}{c}\text { Engagement environnemental } \\
\text { Faible }\end{array}$ & Fort & $\begin{array}{c}\text { Niveau de } \\
\text { signification }\left(\boldsymbol{p}^{\mathbf{a}}\right)\end{array}$ \\
\hline $\begin{array}{l}\text { R\&D effectuée par votre } \\
\text { organisation }\end{array}$ & 2.65 & 2.38 & 2.91 & 0.021 & $* *$ \\
$\begin{array}{l}\text { Communication avec } \\
\text { laboratoires de recherche et } \\
\text { universités }\end{array}$ & 2.22 & 1.91 & 2.49 & 0.004 & $* * *$ \\
$\begin{array}{l}\text { Communication avec des } \\
\text { associations industrielles }\end{array}$ & 2.54 & 2.19 & 2.87 & 0.000 & $* * * *$ \\
$\begin{array}{l}\text { Communication avec } \\
\text { consultants }\end{array}$ & 2.71 & 2.21 & 3.17 & 0.000 & $* * * *$ \\
$\begin{array}{l}\text { Communication avec agences } \\
\text { gouvernementales }\end{array}$ & 2.80 & 2.42 & 3.15 & 0.000 & $* * * *$ \\
$\begin{array}{l}\text { Suivi des changements du } \\
\text { marché }\end{array}$ & 3.14 & 3.06 & 3.21 & 0.405 & \\
$\begin{array}{l}\text { Suivi des changements } \\
\text { technologiques }\end{array}$ & 3.16 & 2.96 & 3.34 & 0.055 & $*$ \\
$\begin{array}{l}\text { Communication avec vos } \\
\text { clients }\end{array}$ & 3.27 & 3.17 & 3.36 & 0.348 & \\
$\begin{array}{l}\text { Communication avec } \\
\text { concurrents }\end{array}$ & 2.66 & 2.44 & 2.87 & 0.057 & $* *$ \\
$\begin{array}{l}\text { Communication avec } \\
\text { fournisseurs }\end{array}$ & 3.20 & 3.04 & 3.34 & 0.140 & \\
\hline a. Niveau de signification: *p<0.10; $* * p<0.05 ; * * * p<0.01 ; * * * * p<0.001$ & & & & \\
\hline
\end{tabular}

\subsubsection{Obstacles}

Les résultats sur l'ensemble de l'échantillon (tableau 10) révèlent que les coûts élevés (3.26) et le temps et l'effort consacrés à l'engagement environnemental (3.24) sont les principaux obstacles perçus par les dirigeants de PME à un plus grand engagement environnemental de leur part. Ce résultat ne surprend pas, considérant que ces raisons sont souvent citées dans la littérature pour justifier de ne pas se préoccuper des problèmes environnementaux ${ }^{8,9,17}$. 
Le fait d'avoir une attitude favorable envers l'environnement ne se traduit pas nécessairement par des actions concrètes. Les dirigeants de PME ne sont peut-être pas au courant de l'impact de leur entreprise sur l'environnement.

De plus, la possibilité d'un manque d'encouragement ou d'incitatif réglementaire (2.54) ainsi que l'attitude du management (2.54) semblent être des raisons moins importantes pour justifier l'inaction des dirigeants de PME vers un parcours plus vert. Ce dernier point est supporté par une recherche qui a trouvé un lien positif entre l'attitude du management et le niveau d'appui à un engagement environnemental accru'. Cependant, les auteurs de cette recherche mentionnent que le fait d'avoir une attitude favorable envers l'environnement ne se traduit pas nécessairement par des actions concrètes. Les dirigeants de PME ne sont peut-être pas au courant de l'impact de leur entreprise sur l'environnement ou encore de ce qu'ils doivent faire pour tenter de l'améliorer. Ces deux derniers éléments sont confirmés par une étude citée dans l'Observatoire des PME européennes de 2002 «Les PME européennes et les responsabilités sociale et environnementale ${ }^{29}$.

Par ailleurs, le niveau d'engagement environnemental poursuivi par les PME influence également le choix des obstacles à un plus grand engagement environnemental. Par exemple, les obstacles où l'on remarque les plus fortes différences observées entre les entreprises à faible engagement environnemental et à fort engagement environnemental sont le manque de sensibilisation et de connaissances de la part des dirigeants (3.32 versus 2.58, $\mathrm{p}<0.001$ ) et la perception de retombées minimes sur l'investissement à court terme (3.34 versus $2.88, p<0.05)$. Ces résultats sont cohérents avec ceux relevés dans la littérature dans la mesure où les études sur les PME ont trouvé que le manque de connaissances des problèmes environnementaux de la part des dirigeants de PME serait un obstacle clé à l'adoption de pratiques environnementales ${ }^{11,20}$. Quant à la perception de retombées minimes sur l'investissement à court terme, le passage suivant de Roy exprime bien la situation qui prévaut chez les PME en comparaison avec les plus grandes entreprises ${ }^{30}$ :

\section{Le niveau d'engagement environnemental poursuivi par les PME influence également le choix des obstacles à un plus grand engagement environnemental. Par exemple, les obstacles où l'on remarque les plus fortes différences observées entre les entreprises à faible engagement environnemental et à fort engagement environnemental sont le manque de sensibilisation et de connaissances de la part des dirigeants.}

«La gestion stratégique des petites entreprises est davantage orientée vers la rentabilité à court terme, alors que la nature inhérente des considérations environnementales exige une vision à long terme. Cette situation créée des barrières importantes à l'adoption d'initiatives environnementales. Dans le cas des plus grandes entreprises, celles-ci bénéficient d'un meilleur accès aux ressources financières et humaines pour intégrer cette dimension dans leurs opérations. » (p. 56)

Pour terminer, l'attitude du management (2.57 versus 2.51), les coûts élevés (3.34 versus 3.19) et la nécessité d'une documentation additionnelle $(3.19$ versus 3,06$)$ sont les trois obstacles pour lesquels l'on retrouve le moins de différences entre les deux groupes.

«La gestion stratégique des petites entreprises est davantage orientée vers la rentabilité à court terme, alors que la nature inhérente des considérations environnementales exige une vision à long terme. Cette situation créée des barrières importantes à l'adoption d'initiatives environnementales. Dans le cas des plus grandes entreprises, celles-ci bénéficient d'un meilleur accès aux ressources financières et humaines pour intégrer cette dimension dans leurs opérations. " 
Tableau 10 - Obstacles à un plus grand engagement environnemental

\begin{tabular}{|c|c|c|c|c|c|}
\hline \multirow{3}{*}{ Coûts élevés } & \multirow{3}{*}{$\begin{array}{c}\begin{array}{c}\text { Moyenne } \\
\text { Échantillon }\end{array} \\
3.26\end{array}$} & \multicolumn{2}{|c|}{ Engagement environnemental } & \multirow{2}{*}{\multicolumn{2}{|c|}{$\begin{array}{c}\text { Niveau de } \\
\text { signification }\left(p^{\mathrm{a}}\right)\end{array}$}} \\
\hline & & \multirow{2}{*}{$\begin{array}{c}\text { Faible } \\
3.34\end{array}$} & \multirow{2}{*}{$\begin{array}{l}\text { Fort } \\
3.19\end{array}$} & & \\
\hline & & & & 0.499 & \\
\hline $\begin{array}{l}\text { Nécessite trop de temps et } \\
\text { d'effort }\end{array}$ & 3.24 & 3.43 & 3.08 & 0.075 & $*$ \\
\hline $\begin{array}{l}\text { Manque de sensibilisation et de } \\
\text { connaissances de la part des } \\
\text { dirigeants }\end{array}$ & 2.93 & 3.32 & 2.58 & 0.001 & $* * * *$ \\
\hline $\begin{array}{l}\text { Perception qu'un expert est } \\
\text { requis et que le coût associé est } \\
\text { trop élevé }\end{array}$ & 3.07 & 3.30 & 2.87 & 0.057 & $*$ \\
\hline $\begin{array}{l}\text { Perception de retombées } \\
\text { minimes sur l'investissement à } \\
\text { court terme }\end{array}$ & 3.10 & 3.34 & 2.88 & 0.037 & $* *$ \\
\hline $\begin{array}{l}\text { Nécessite des contrôles } \\
\text { additionnels }\end{array}$ & 3.08 & 3.22 & 2.96 & 0.224 & \\
\hline $\begin{array}{l}\text { Nécessite de la documentation } \\
\text { additionnelle }\end{array}$ & 3.12 & 3.19 & 3.06 & 0.519 & \\
\hline $\begin{array}{l}\text { Peur, hostilité et crainte } \\
\text { associées avec l'attitude du } \\
\text { management face à des } \\
\text { pratiques d'affaires plus } \\
\text { respectueuses de } \\
\text { l'environnement }\end{array}$ & 2.54 & 2.57 & 2.51 & 0.773 & \\
\hline $\begin{array}{l}\text { Aucun encouragement ou } \\
\text { incitatif réglementaire }\end{array}$ & 2.54 & 2.72 & 2.38 & 0.085 & $*$ \\
\hline
\end{tabular}

\subsubsection{Pratiques écologiques}

En dernier lieu, à l'aide d'une question ouverte, nous avons tenté de recueillir les pratiques écologiques les plus souvent utilisées par les PME agroalimentaires (voir tableau 11). Les trois activités les plus citées sont la réduction du gaspillage et des déchets (14), les programmes de recyclage (10) et l'utilisation de matériaux recyclés (10). Une étude comparative ${ }^{17}$ effectuée en Allemagne, en Italie et au Royaume-Uni révèle des résultats similaires où la réduction du gaspillage et l'utilisation de matériaux recyclés furent les deux premiers choix des 97 entreprises interrogées. Les résultats obtenus confirment également ceux de (Berger-Douce, 2007) dans la mesure où les 84
PME françaises interrogées ont privilégié des activités environnementales comme le recyclage des déchets $(91,7 \%)$ et la réduction du gaspillage $(70,2 \%)$.

Nos résultats révèlent également des méthodes simples comme la minimisation du papier par le courriel jusqu'à des méthodes plus dispendieuses et plus ambitieuses comme l'achat de nouvel équipement moins énergivore. D'autres pratiques utilisées sont l'utilisation de matériaux recyclés, recyclables ou biodégradables, la conservation de l'énergie et de l'eau, la gestion des eaux usées et un meilleur dosage des produits de nettoyage. 
Tableau 11 - Description des pratiques écologiques utilisées par les PME

-Réduction du gaspillage, des déchets, de l'emballage et des sacs de plastiques

-Programme de recyclage

-Procédures d'affaires écologiques (ex. courriel, sensibilisation des clients, etc.)

-Compostage de déchets organiques

-Utilisation de matériaux recyclés, recyclables ou biodégradables

-Nouvelle technologie/Nouvel équipement

-Gestion des eaux usées

-Conservation de l'énergie et de l'eau

\section{CONCLUSION}



Globalement, cette étude visait à acquérir une meilleure compréhension de l'engagement environnemental au sein de PME qui œuvrent dans le domaine agroalimentaire. Plus spécifiquement, les objectifs de cette recherche étaient de voir si certains facteurs internes étaient susceptibles de favoriser l'engagement environnemental et d'identifier les obstacles qui empêchent les dirigeants de PME de s'engager davantage en matière d'environnement.

Premièrement, très peu de différences ont été observées dans l'analyse des résultats comparatifs entre les trois pays. Il est tout de même intéressant de constater que les PME du Canada démontrent un niveau d'engagement qui se situe entre celui de la France et celui de la Finlande, ce qui laisse supposer que les dirigeants de PME canadiennes valorisent autant l'environnement que ceux des pays européens.

Deuxièmement, la création de deux groupes de références (faible engagement environnemental et fort engagement environnemental) s'est avérée très révélatrice pour évaluer la stratégie environnementale des PME dans la mesure où les PME avec un fort engagement environnemental ont obtenu des résultats statistiquement significatifs $(p<0.001)$ pour chacun des quatre énoncés de l'échelle et, également dans le cas où les quatre énoncés ont été regroupés en un seul facteur.

Troisièmement, les résultats suggèrent que la situation financière, l'âge et la taille de l'entreprise n'ont pas d'impact significatif sur l'engagement environnemental. Bien que le score obtenu sur chacune de ces variables ait été supérieur dans le cas des PME avec un fort engagement environnemental, il ne nous est pas permis de considérer ces variables comme déterminantes de l'engagement environnemental.

Quatrièmement, l'étude révèle que les PME démontrant un engagement environnemental fort possèdent des compétences liées à l'innovation et qu'elles consacrent surtout plus de ressources à l'innovation sur les produits et à la recherche et développement. Les résultats suggèrent également que les PME de l'ensemble de l'échantillon consacrent beaucoup d'efforts aux activités d'acquisition de connaissances, particulièrement auprès de leurs clients et de leurs fournisseurs tandis que les PME démontrant un engagement environnemental élevé consacrent plus d'efforts aux activités de communication avec les agences gouvernementales, les associations industrielles et les consultants $(\mathrm{p}<0.001)$. 
Finalement, les coûts élevés et le temps et l'effort consacrés à l'engagement environnemental sont les deux principaux obstacles cités par les dirigeants de PME à un engagement environnemental plus fort.

Notre étude comporte certaines limites. En effet, compte tenu de la nature restreinte de l'échantillon (101 PME) et de la limite liée aux instruments de mesure utilisés qui représentent des mesures perceptuelles et non une évaluation objective, il nous est impossible de dégager des conclusions pouvant être généralisées à l'ensemble des PME.

Quant aux recherches futures, nos résultats indiquent que le manque de sensibilisation et de connaissances de la part des dirigeants est l'obstacle majeur qui différencie le plus les PME à faible engagement environnemental de celles à fort engagement environnemental. Il serait donc judicieux de porter une attention particulière à cet obstacle dans le but de développer une meilleure compréhension des obstacles qui freinent un plus grand engagement environnemental. À ce sujet, il faudrait offrir aux dirigeants de PME des programmes de sensibilisation et d'éducation afin de pallier leur manque d'expertise et de connaissances ${ }^{30}$. Ainsi, une telle recherche permettrait de répondre à des questions telles que : quelles sont les organisations les mieux placées pour offrir ce genre de service, de quel type d'information (technique, scientifique ou managériale) les dirigeants de PME ont-ils besoin, combien sontils prêts à payer, etc.

\section{Le manque de sensibilisation et de connaissances de la part des dirigeants est l'obstacle majeur qui différencie le plus les PME à faible engagement environnemental de celles à fort engagement environnemental.}

\section{BIBLIOGRAPHIE}

${ }^{1}$ Robbins, P. T. (2001). Greening the Corporation: Management Strategy and the Environmental Challenge, Londres, Royaume-Uni: Earthscan Publications.

${ }^{2}$ Hillary, R. (2000). Small and Medium-sized Enterprises and the Environment, Sheffield, Royaume-Uni: Greenleaf Publishing.
${ }^{3}$ Schaper, M. (2002). Small Firms and Environmental Management, International Small Business Journal, 20: 3, 235-251p.

${ }^{4}$ Commission Européenne, Entreprises et industrie. (2009), Small Business Act (SBA) : Fiche technique de la Finlande et de la France - 2009. Consulté le 7 septembre 2010.

${ }^{5}$ Environnement Canada. (2010). Petites et moyennes entreprises, Consulté le 7 septembre 2010, http://www.ec.gc.ca/p2/default.asp?lang=Fr\&n=B49 AE71A-1

${ }^{6}$ Roy, M. J., S. Berger-Douce, et J. Audet (2008). L'engagement environnemental en PME : l'influence des ressources, des compétences et des connaissances, Journal of Small Business and Entrepreneurship, 21: 1, 75-94 p.

${ }^{7}$ McKeiver, K., et D. Gadenne (2005). Environmental Management Systems in Small and Medium Businesses, International Small Business Journal, 23(5), 513-537 p.

${ }^{8}$ Revell, A., D. Stokes, et H. Chen (2010). Small Businesses and the Environment: Turning Over a New Leaf? Business Strategy and the Environment, 19, 273-288 p.

${ }^{9}$ Gadenne, D.L., J. Kennedy, et C. McKeiver (2009). An Empirical Study of Environmental Awareness and Practices in SMEs, Journal of Business Ethics, 84:1, 45-63 p.

${ }^{10}$ Berger-Douce, S. (2007). Le management environnemental des PME rentables: Une étude exploratoire en France, Revue internationale PME, $20: 3-4,165-190$ p.

${ }^{11}$ Roy, M.J., et F. Thérin (2008). Knowledge Acquisition and Environmental Commitment in SMEs. Corporate Social Responsibility and Environmental Management, 15, 249-259 p.

${ }^{12}$ Tilson, N. (2007). SMEs Getting Green Message. September 26, Belfast Telegraph Newspapers Ltd.

${ }^{13}$ Gunningham, N., D. Sinclair et P. Burritt (1997). Barriers and Motivators to the Adoption of Cleaner Production Practices, ACEL Final Report, Environment, Australia, Australian Centre for Environmental Law and the Australian National University.

${ }^{14}$ Spence, L.J, R. Rutherford, et R. A. Blackburn (1998). Small Business and Environmental Issues in the UK and the Netherlands: A Literature review and research agenda. Kingston. Upon Thames, Surrey, Royaume-Uni: Small Business Research Centre, Kingston University.

${ }^{15}$ Rutherfoord, R., R.A. Blackburn, et L.J. Spence. (2000). Environmental Management and the Small Firm: An International Comparison. International Journal of Entrepreneurial Behaviour and Research, 6: 6, 310-325 p. 
${ }^{16}$ Berger-Douce, S. (2006). Les enjeux stratégiques de l'engagement environnemental des petites entreprises. Gestion 2000, janvier-février, 171-188 p.

${ }^{17}$ Hitchens, D., J. Clausen, M. Trainor, M. Keil et S. Thankappan. (2003). Competitiveness, Environmental Performance and Management of SMEs. Greener Management International, 44, 45-57 p.

${ }^{18}$ Welsh, J. A., et J. F. White. (1981). A Small Business is not a Big Business. Harvard Business Review, 59: 4, 18-32 p.

${ }^{19}$ Rowe, J., et D. Hollingsworth. (1996). Improving Environmental Performance of SMEs. EcoManagement and Auditing, 3, 97-107 p.

${ }^{20}$ Hitchens, D., S. Thankappan, M. Trainor, J. Clausen et B. De Marchi. (2005). Environmental Performance, Competitiveness and Management of Small Business in Europe. Tijdschrift voor Economische En Sociale Geografie, 96: 5, 541-557 p.

${ }^{21}$ Smith, A., R. Kemp, et C. Duff. (2000). Small Firms and the Environment: Factors that Influence Small and Medium-sized Enterprises Environmental Behaviour. Dans R. Hillary (dir.), Small and Medium-sized Enterprises and the Environment: Business Imperatives, Sheffield, Royaume-Uni: Greenleaf Publishing.

${ }^{22}$ Howarth, R., et K. Melton. (2001). Environmental Business Support for SME's: the Marketing Factor. The 2001 Eco-Management and Auditing Conference and Research Workshop on Corporate Environmental Management, Nijmegen, the Netherlands.

${ }^{23}$ Perez-Sanchez, D., J.R. Barton, et D. Bower. (2003). Implementing Environmental Management in SMEs. Corporate Social Responsibility and Environment Management, 10, 67-77 p.

${ }^{24}$ Hillary, R. (1999). Evaluation of Study Reports on the Barriers, Opportunities and Drivers for Small and Medium Sized Enterprises in the Adoption of Environmental Management Systems. Report Submitted to Department of Trade and Industry Environment Directorate, London.

${ }^{25}$ Cragg, P.B., et M. King. (1988). Organizational Characteristics and Small Firms Performance Revisite. Entrepreneurship Theory and Practice, 13: 2, 49-63 p.

${ }^{26}$ Macpherson, A., et A. Wilson. (2003). Enhancing SMEs Capability: Opportunities in Supply Chain Relationship. Journal of Small Business and Enterprise Development, 10:2, 167-179 p.

${ }^{27}$ Nunally, J.C., et I. H. Bernstein. (1994). Psychometric Theory. Third Edition, New York: McGraw-Hill.

${ }^{28}$ Terlier, A. (2009). Le développement durable dans les PMI de l'agroalimentaire. Centre Régional d'Observation du Commerce de l'Industrie et des Services (CROCIS), 4 p. Consulté en octobre 2011. Disponible sur www.crocis.ccip.fr/telecharger-151documents-agroalimentaire.pdf
${ }^{29}$ Commission Européenne, DG Entreprise. (2002). Les PME européennes et les responsabilités sociale et environnementale. Rapport de l'Observatoire des PME européennes 2002, No 4, 56 p.+ annexes. http://ec.europa.eu/enterprise/policies/sme/facts-figures-analysis/performance-review/pdf/final/ sba_fact sheet_finland_en.pdf; http://ec.europa.eu/enterprise/ policies/sme/facts-figures-analysis/performance-

review/pdf/final-

vo/sba_fact_sheet_fr_french_100525.pdf

${ }^{30}$ Roy, M.J. (2005). Les défis de la mise en ouvre d'une stratégie environnementale. Revue Organisations et territoires, Hiver 2005, 55-61 p.

\section{NOTES}

${ }^{a}$ Le secteur agroalimentaire a été retenu parce que c'est un secteur commun à chacun des pays participant à l'étude et dans lequel plusieurs PME opèrent.

${ }^{\mathrm{b}}$ Les 3 pays participant à l'étude sont membres d'un consortium international sur la gestion de l'environnement. Ce consortium regroupe des institutions d'enseignement du Canada, de la Finlande, de la France, et des Pays-Bas. L'objectif premier de ce consortium est de sensibiliser les étudiants à la gestion environnementale.

${ }^{\mathrm{c}}$ Seuls les résultats statistiquement significatifs sont présentés afin d'alléger le texte. 